This is an electronic reprint of the original article. This reprint may differ from the original in pagination and typographic detail.

Author(s): Chang, Zheng; Zhu, Kun; Zhou, Zhenyu; Ristaniemi, Tapani

Title: $\quad$ Service Provisioning with Multiple Service Providers in 5G Ultra-dense Small Cell Networks

Year: $\quad 2015$

Version:

Please cite the original version:

Chang, Z., Zhu, K., Zhou, Z., \& Ristaniemi, T. (2015). Service Provisioning with Multiple Service Providers in $5 G$ Ultra-dense Small Cell Networks. In K.-C. Leung (Ed.), Proceedings of IEEE PIMRC 2015 : IEEE 26th International Symposium on Personal, Indoor and Mobile Radio Communications (pp. 1895-1900). Institute of Electrical and Electronic Engineers. IEEE International Symposium on Personal, Indoor, and Mobile Radio Communications workshops. https://doi.org/10.1109/PIMRC.2015.7343608

All material supplied via JYX is protected by copyright and other intellectual property rights, and duplication or sale of all or part of any of the repository collections is not permitted, except that material may be duplicated by you for your research use or educational purposes in electronic or print form. You must obtain permission for any other use. Electronic or print copies may not be offered, whether for sale or otherwise to anyone who is not an authorised user. 


\title{
Service Provisioning with Multiple Service Providers in 5G Ultra-dense Small Cell Networks
}

\author{
Zheng Chang*, Kun Zhu ${ }^{\dagger}$, Zhenyu Zhou ${ }^{\ddagger}$, Ekram Hossain ${ }^{\dagger}$, Tapani Ristaniemi* \\ ${ }^{*}$ University of Jyvaskyla, Department of Mathematical Information Technology, P.O.Box 35, FI-40014 Jyvaskyla, Finland \\ $\dagger$ Department of Electrical and Computer Engineering University of Manitoba, Winnipeg, MB, Canada \\ $\ddagger$ State Key Laboratory of Alternate Electrical Power System with Renewable Energy Sources, \\ School of Electrical and Electronic Engineering, North China Electric Power University, Beijing 102206, China \\ Email: zheng.chang@jyu.fi
}

\begin{abstract}
In this work, a three-stage game theoretical approach for addressing the virtual network service providers (NSPs), small cell provider (SCP) and user interaction in heterogenous small cell networks is presented. In particular, we consider the users can select the services of different NSPs based on their prices. The NSPs have no dedicated hardware and need to rent from the SCP in term of radio resources, e.g., small cell base stations (SBSs) in order to provide satisfied services to the users. Due to the fact that the selfish parties involved aim at maximizing their own profits, a hierarchical dynamic game framework is presented to address interactive decision problem. In the lower-level a Stackelberg game is formulated to model and analyze the adaptive service selection of non-atomic users. In the upper-level, the NSPs and SCP sequentially determine the leasing and pricing strategies, respectively, by taking into account the service selection in the lower-level game. Performance evaluation shows the effectiveness and advantages of the proposed game theoretic approaches.
\end{abstract}

Index Terms-non-atomic, small cell networks; game theory; heterogenous services; pricing; competition;

\section{INTRODUCTION}

The explosive growth of the smartphone industry, multimedia services and mobile applications heavily relies on the high speed data rate transmission. Meanwhile, although the rapidly-increasing high speed wireless networks are continually coloring our life and even changing our daily behaviors, the demand for high data rate services is straining the current network much faster than before. Moreover, to acquire the data services, the connection between users and base station (BS) also urge the development of the future wireless networks. One promising path towards a high speed data rate next generation network is to deploy low cost, low power small cell (SC), including Picocell, Femtocell, or even WiFi network to assist the transmission within the macrocell [1].

The recent emerging mobile platforms, such as software defined network (SDN) and cloud radio access networks (CRANs), bring us new thoughts and views on the current cellular wireless networks, which urge to rethink the current infrastructure. The recent advances also open the door to the extension of SDN/network function visualization (NFV) concepts to RAN, creating thus the Virtual RAN (VRAN) concept where the execution of RAN functions is moved from dedicated telecom hardware to commoditized platforms.
Leveraging such concept, a VRAN operator does not necessarily has its own BS. Instead, it can rent the BSs whenever needed, which is able to reduce its operation cost. Meanwhile, as small cell BSs (SBSs) are going to be deployed with high density in the future $5 \mathrm{G}$ networks [2], leasing SBSs instead of building might be energy and cost efficient for all kind of network providers. Moreover, even if a RAN operator is interested in having its own dedicated platforms, it can still lease the resources to other VRANs if available to make more profits. On the other hand, the dedicated small cell provider (SCP) can focus on the maintenance of SBS without distracting itself on the network pricing, operation and advertising. Moreover, it can also coordinate the SBS deployment so that less resources, such as, pipes, towers, electronics, land, can be wasted, which is considered to be friendly to the environment. Such kind of flexible network architecture is also able to lower the entrance standard of proving network services, which apparently will result in a network with diverse and abundant services. Therefore, in this paper, we consider a scenario where multiple network service providers (NSPs), (i.e. VRAN operators) aim at selling network services to multiple users who are free to choose provider(s) and services. Under the assumption that all SBSs are managed by a small cell provider (SCP), NSPs also need to compete for renting (all or parts of) SBSs from SCP. We first investigate the problem that how NSPs set prices for the services and how users purchase services. Then we concentrate on how the SCP set the price for resource leasing and how the NSPs react and compete with each other.

Applying game theory to solve the network service selection problem has already drawn much attention during the last decade. In [3], authors presented a game theoretic approaches for the service selection in present of different RANs and each of them provided two types of services. Authors of [4] proposed an auction based model that can be used to dynamically allocate the spectrum to service providers and study the service pricing mechanisms that the providers can use. A Stackelberg game is formulated in [5] to capture the interaction between service providers and users. When considering SC (Femtocell) as one complementary network element, most of the work is dedicated to study how to allocate the spectrum to the SCs. In [6], a spectrum leasing method that 
allowing SBS to rent spectrum from the coexisting macrocell was presented. In order to encourage SCs to accommodate macrocell users, a refund mechanism was proposed in [7]. Authors of [8] focused on the SC market and studied entrant NSPŠs long-term decision about whether to enter the market and which spectrum sharing technology to select to maximize considered profit. To study the resource allocation scheme in a SCs underlaid macrocell, the work of [9] concentrated on a Stackelberg game that is formulated to study the joint utility maximization of the macrocell and the SCs subject to an interference power constraint. In [10], authors presented a dynamic evolutionary game approach to study user's equilibria and a Stackelberg game to model to analyze the interaction between NSP and SCPs. Most of the previous works considered individual user in the network as the game player and correspondingly analyze its strategy and equilibria. However, in reality, individual choice of user does not usually affect neither the system performance nor the strategy of NSPs. On the other hand, the aggregate behaviors or the choices of many users in the same subject do have serious impact on them, where the users are referred as non-atomic users. It can be observed that only minority of the works, e.g., [5] [8] studied it under the interaction of NSP and users. Therefore, in this work, our focus is to study the decision/price making strategies in present of non-atomic users, multiple NSPs and services, and one SCP. Comparing with previous existed works, the main contributions of this work can be summarized as follows:

- We propose a novel model to describe and study the SC networks with SCP, competitive NSPs and a large number of users. A three-stage hierarchical (two-level) novel game framework is formulated with objective to find the equilibria point of users, NSPs, and SCP.

- The competition among NSPs are explicitly studied. In the upper-level, a model for competitive SBS leasing among NSPs in heterogenous small cell is proposed and solved based on the noncooperative game model;

- In the lower-level, when considering non-atomic users, we first focus on the interaction among users and service selection of users and formulate a multi-leader multifollower Stackelberg game. When the user behavior is known, then the optimal pricing strategies of NSPs is presented to obtain the Stackelberg equilibrium (SE).

- The performance of the our proposed schemes is evaluated under different system parameters. Extensive simulations are carried out to illustrate the advantages of this work.

The rest of this paper is organized as follows. Section II presents system model and game framework. In Section III, the lower-level game consisting of users and NSPs is studied. In Section IV, we investigate the strategies of NSPs and SCP in the upper-level game. The benefits of our proposed algorithm is demonstrated in Section $\mathrm{V}$ through performance evaluations and finally we conclude this work in Section VI.

\section{SySTEM MODEL}

\section{A. Network Model}

We consider there is a SCP in the network and the SCP has many SBSs that are deployed with ultra-density, which is able to provide sufficient coverage to a large number of users. The SCP is able to lease its resources, such as SBSs, or part of SBSs (in term of spectrum), to different NSPs. It is also assumed that there are $K$ network service providers (NSPs), whose set is $\mathcal{K}:=\{1,2, \ldots, K\}, K>1$ and $N$ users, whose set is $\mathcal{N}:=\{1,2, \ldots, N\}, N>>1$. The set of services offered by NSP $k$ is denoted as $\mathcal{S}_{k}:=\left\{1,2, \ldots, S_{k}\right\}$. Accordingly, each offered service can be presented by a pair $(k, s)$, where $k \in \mathcal{K}, s \in \mathcal{S}_{k}$. We can define a vector $y$ describing the service-NSP pair $(k, s)$, where $y \in \mathcal{Y}:=\{1,2, \ldots, Y\}, Y=$ $\sum_{k=1}^{K}\left|\mathcal{S}_{k}\right|$.

In the system, $N$ users can freely choose their services offered by different NSPs and the NSPs select service pricing strategies to maximize their own payoffs. We also consider the market of SCs is a monopoly market. Therefore, the NSPs need to lease radio resources, i.e., SBS and associated spectrum, from one SCP. The SCP selects its optimal pricing strategies for maximizing its payoff and the NSPs decide their leasing policies based on SCP's price and the users' choices of services. Such kind of flexible network architecture is also able to lower the entrance standard of proving network services, which apparently will result in a network with diverse and abundant services.

\section{B. Two-level Game Framework}

The formulated hierarchical game structure is presented in Fig. 1. In our proposed game, the players are categorized into two levels. In the lower-level, users and NSPs form a multi-leaders multi-followers Stackelberg game. NSPs are the Stackelberg leaders who set the prices for the provided services, and announces the information to the users who are the Stackelberg followers. The users are non-atomic so that the strategic interaction among the users is considered to be extremely poor due to its great number. More precisely, individual choices in the game is almost nil, while aggregated behavior of a number of users is relevant. This is a practical setting since a single user can not consider his choice affect the others and in the reality, the choice of individual user is not able to affect the strategy of service provider, but the choices of many users can. In the upper-level, NSPs and SCP are the players in the game. The outcomes of both levels have impacts on each other. On one hand, the pricing strategies of SCP affect the NSPs' pricing strategies in the lower-level game. On the other hand, the choices of user in the lower-level game also have impact on the NSPs' leasing policies in the upper-level game. The payoffs of the NSPs depend on the behaviors of users and SCP, as well as their own pricing strategies.

\section{Lower-LeVel GAme: Service Provisioning AND ACQUISITION}

The lower-level game which describes the service provisioning and acquisition process consists of two stages, of 


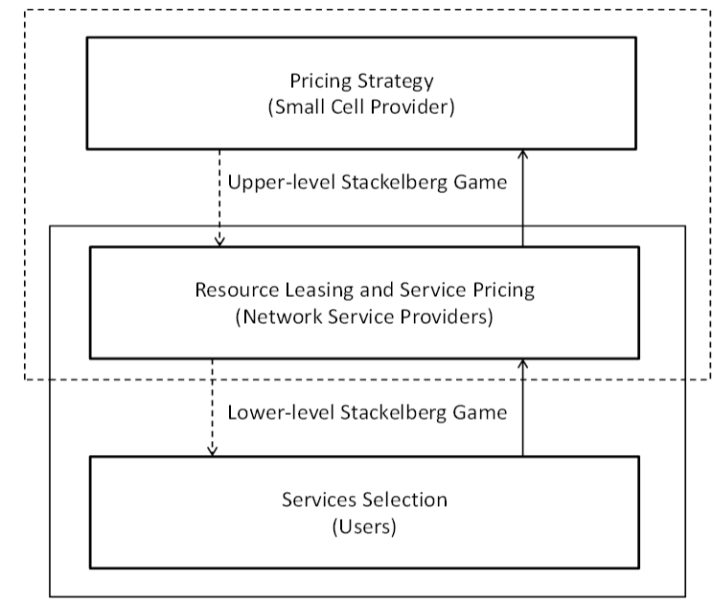

Fig. 1. Framework of Formulated Game

which the first one is that NSPs announces their services. The corresponding prices associated with the services are chosen to maximize NSP's revenue. In the second stage, users choose the services and NSPs based on the the prices and their needs, i.e. data rates. We consider one user can only select the service of single NSP at one time. To obtain the best payoff, the users can periodically adapt the service selections according to changes in prices or QoS. The NSP will consider the users' choices and accordingly, choose the SBS leasing policies and update the prices of the services if needed to maximize their revenues. As such, we describe the interaction between user and NSP as a Stackelberg game, which consists of a follower's game and a leader's game. In the followings, we first introduce the payoff models of users and NSPs, and present the game formulation correspondingly. Then we analyze the formulated multi-leader multi-follower Stackelberg game with details.

\section{A. Game Formulation for Users and NSPs}

1) Payoff Model of Users: The users consider the network price vectors $\mathcal{P}:=\left\{p_{1}, p_{2}, \ldots, p_{Y}\right\}$ as a set of pricing constants and aim at choosing a service that maximizes their own satisfactions. We define $\alpha$ as the user's valuation for the QoS. To this point, we define the payoff function of users selecting service pair $y$ as

$$
U_{y}\left(d_{y}\right)=\alpha Q_{y}\left(d_{y}\right)-p_{y},
$$

where $Q_{y}($.$) is the utility/reward, e.g., QoS, that a user$ can preserve when choosing pair $y . p_{y}$ is the price vector of $y$ and we assume $\mathcal{P}$ is in an ascendent order. $\alpha$ is the associated factor to link the QoS of service to the cost $p_{y}$. We denote $d_{y}$ as the fraction of users selected service $y$ and $\boldsymbol{d}:=\left(d_{1}, d_{2}, \ldots, d_{Y}\right)$ as the user distributions or load profile. Without loss of generality, we assume that $Q_{y}\left(d_{y}\right)$ is continuous, differentiable and monotonous non-increasing non-negative function of $d_{y}$, which is a common assumption in [5] [8].

Since we consider a continuum of users, the competition among users is modeled as a non-atomic game
$\mathfrak{G}_{f}\left(\mathcal{N}, \mathcal{P},\left(U_{y}\right)_{y \in \mathcal{Y}}\right)$. The users in $\mathcal{N}$ are non-atomic players, $\mathcal{P}$ is the price set, and $\left(U_{y}\right)_{y \in \mathcal{Y}}$ is the set of payoff functions. The users in this game consider the prices of services are independent from their choices and are only decided by the NSPs. In general, on each time the users will select the services with assumption that prices are fixed.

2) Payoff of NSPs: Each NSP $k \in \mathcal{K}$, can autonomously select its price vector, $\boldsymbol{p}_{k}:=\left\{p_{y} \mid \forall y \in \mathcal{S}_{k}\right\}$, so as to maximize its own revenue. The main difference between leaders and followers, in a hierarchical game, is that the leaders can predict the reaction of the followers to their strategies, while the followers are myopic. As opposed to the non-atomic game of users, the leaders' competition is a noncooperative Nash game, which can be expressed in normal-form by $\mathfrak{G}_{f}\left(\mathcal{K},\left(\boldsymbol{p}_{k}\right)_{k \in \mathcal{K}},\left(u_{k}\right)_{k \in \mathcal{K}}\right) . u_{k}$ denotes the payoff of NSP $k$, which is defined as:

$$
u_{k}\left(\boldsymbol{p}_{k}, \boldsymbol{p}_{-k}\right):=\sum_{s \in \mathcal{S}_{k}} N d_{s}^{*}(\mathcal{P}) p_{s}-\Upsilon_{k},
$$

where $\boldsymbol{p}_{-k}$ is the price set of NSPs except $k . \Upsilon_{k}$ is cost of leasing resulted from upper-level game. We use $\Upsilon_{k}$ here to present the fact that when one NSP makes decision on their pricing, the cost is already known and fixed. Hence, $d_{s}^{*}(\mathcal{P})$ represents the fraction of users employing service $s$ when the underlying system is at the equilibrium given the price set $\mathcal{P}$.

\section{B. Analysis of Lower-level Game}

We have presented the payoff functions of both users and NSPs. In the following, we analyze the formulated multi-leader multi-follower Stackelberg game. Using noncooperative game, NSPs will set their prices to maximize individual payoffs and users select services to maximize their utilities correspondingly. A common solution concept in the Stackelberg game is sub-game perfect equilibrium (SPE) and it is known that backward induction method is a powerful tool to obtain SPE. To achieve it, we start with the followers in the game assuming of information of price set. Then the analysis goes back to the leader and study how the leaders choose pricing vectors.

1) User's Game in Lower-level Game: Non-atomic games were originally investigated for addressing the transportation problem [11], where players choose route towards the destination. In a non-atomic game, the interaction among players happens via aggregated action changes: individual decision of players does not significantly affect the payoff, but rather, choice changing of a proportion of players does have impact. In other word, the aggregate data demand of the users subscribing to the NSPs' data plan does not change at the equilibrium. In this work, our considered work involves a large number of users of which the load distribution has impact on the utility performance in (1). Therefore, the game can be modelled within the framework of non-atomic game. The solutions of the non-cooperation behavior in non-atomic game refers to Wardrop equilibrium (WE), which is also known as Wardrop's first principle or user equilibrium in this case. Interpreting Wardrop's first principle, WE in this work can be defined as follows, 
Definition 1. A load distribution $\boldsymbol{d}^{*}$ is a WE in the pure strategy game $\mathfrak{G}_{f}$, iff $\forall y \in \mathcal{Y}$,

$$
U_{y}\left(\boldsymbol{d}^{*}\right)=\max _{z \in \mathcal{Y}} U_{z}\left(\boldsymbol{d}^{*}\right),
$$

In order to study the SE of the considered hierarchical game, we first need to analyze the WE of the user's game. Therefore, our first result pertains the existence of at least one WE in pure strategies for the game $\mathfrak{G}_{f}$ for any arbitrary pricing profile, which is stated below:

Theorem 1. In the game $\mathfrak{G}_{f}$, if the following conditions are met, for any prices in $\mathcal{P}$, there is at least one pure strategy in WE [12].

- 1. The utility functions depend only on the traffic load and not on the users' identities;

- 2. The $Q o S$ functions, i.e., $\boldsymbol{Q}_{y}(\boldsymbol{d})$ are continuous functions of $\boldsymbol{d}, \forall y \in \mathcal{Y}$;

The proof of Theorem 1 follows Theorem 1 and 2 in [12]. It can be well observed that our considered model follows both conditions. Theorem 1 guarantees that there is at least one WE in the follower's game for any price strategies of leader's game. Next we show the uniqueness of the WE in Theorem 2 .

Theorem 2. In the game $\mathfrak{G}_{f}$, iff the following conditions are met, for any prices set $\mathcal{P}$, there is a unique equilibrium [13].

- 1. The utility functions is strictly monotone;

- 2. There exists a Wardrop equilibrium.

The proof of Theorem 2 can be derived from [14]. It is also indicated that WE is identical to a convex optimization problem when the assumptions are satisfied. Therefore, a unique WE solution exists. The convergence of the game with non-atomic users has been discussed in [8] [5]. In particular, a Krasnoselskii algorithm [15] is proposed in [5] and it has been proved that through Krasnoselskii algorithm, WE can be reached.

2) NSP's Game in Lower-level Game: In this part, we present the analysis on the NSP's game. In our formulated Stackelberg game, the NSPs need to select the pricing policies based on the service selection behavior of users to maximize their payoffs. To this point, one can observe that NSP's game is a Nash game, and a suitable solution is the Nash equilibrium (NE), which is a set of pricing vector such that no NSP can gain by unilaterally modifying its own strategy. We can have the following definition for NE,

Definition 2. A price vector $\boldsymbol{p}^{*}$ is a NE in game $\mathfrak{G}_{l}$, if $\forall k \in \mathcal{K}$,

$$
u_{k}\left(\boldsymbol{p}_{k}^{*}, \boldsymbol{p}_{-k}\right) \geq u_{k}\left(\boldsymbol{p}_{k}, \boldsymbol{p}_{-k}\right)
$$

In this case, NE is obtained by using best response function which is the best strategy that one NSP can select to maximize its own utility given others' choices. We have the best response function defined as

$$
\mathcal{B}\left(\boldsymbol{d}\left(\boldsymbol{p}_{-k}\right)\right)=\arg \max _{\boldsymbol{p}_{k}} \sum_{s \in \mathcal{S}_{k}} N d_{s}^{*}\left(\boldsymbol{p}_{k}, \boldsymbol{p}_{-k}\right) p_{s} .
$$

The set $\mathcal{P}$ denotes the NE of NSP's game, iff for any $\boldsymbol{p}_{k} \in \mathcal{P}$

$$
\boldsymbol{p}_{k}=\mathcal{B}\left(\boldsymbol{d}^{*}\left(\boldsymbol{p}_{-k}\right)\right) .
$$

(4) indicates that the best pricing strategy of NSP $k$ is the one that maximizes its own payoff given other NSPs' strategies. It is reasonable to consider that the NSP has information of user distribution or load profile at a certain time, and users rationally update their choices. Thus, NSPs is able to predict the user's load at WE. In the studied hierarchical game, the SPE in leader's game represents the state in which no user can achieve a higher payoff by switching service, and no leader can obtain higher revenue by changing its pricing strategy. Generally, the SE for a Stackelberg game can be obtained by finding its SPE. To investigate SE of the studied hierarchical game, we can then study the NE in the leader's game. With the assumption that $\boldsymbol{d}^{*}$ is reached by that fact that at each time, fraction of users rationally updates its choice (i.e., users behavior according to Krasnoselskii algorithm), we have the following theorem:

Theorem 3. In the game $\mathfrak{G}_{l}$, there exists at least one $N E$.

Proof. To prove Theorem 3, we need to show the following conditions are satisfied,

- 1. $\left\{p_{1}, p_{2}, \ldots p_{Y}\right\}$ is a nonempty, convex, and compact subset of Euclidean space ;

- 2. $\boldsymbol{d}^{*}($.$) is a continues function of price \mathcal{P}$.

It can be well observed that our model satisfies the first condition. The fulfillment of the second condition can be derived from Theorem 11 in [5]. Thus, game $\mathfrak{G}_{l}$ has at least one NE.

\section{UPPER-LEVEL GAME: RESOURCE LEASING AND PRICING}

In the upper-level game, the NSPs need to lease radio resources, e.g., frequency spectrum of SBS, from SCP with consideration of the service selection of users, while SCPs need to make the optimal decisions on the pricing strategy. For each NSP, increasing the price of leasing could obtain more resources from SCP and the QoS offered by the NSP improves. However, the expense also increases which may result in a reduced total payoff of the NSP. Moreover, the NSP may consider to increase its pricing of services which also lead to a decreased number of subscribers and service satisfaction. For the SCP, increasing the price could bring more payoffs. However, it may also come with some disadvantages such as low leasing ratio of SBSs, etc. In particular, we consider SCP have the same pricing over its resources. To interpret the resource competition and pricing procedure, the strategy of NSP is the requested spectrum size in SCs. The ultra-dense nature of SBS deployment can ensure there are enough SBSs to serve the users, and SCP can then decide which SBSs are used as data transmitter. To address such dynamic interactive decision making problem, we formulate a Stackelberg game in which the SCP and NSPs are the leader and followers, respectively. 


\section{A. Payoff Models of NSP and SCP}

1) Payoff of SCP: In the considered system, SCP can set its price for renting the small cells to the requested NSPs. Or equivalently, SCP can lease the radio resources, such as spectrum bandwidth in a small cell to the NSPs. The cost model of SCP is assumed to consist of operational cost $e_{0} \in \boldsymbol{R}_{+}$,such as a fixed cost independent of workload, and transmission cost $e_{1} \in \boldsymbol{R}_{+}$, such as power consumption and spectrum usage. Thus, the cost model of SCP can be defined as

$$
E(\boldsymbol{e}, \mathbb{C}):=e_{0}+e_{1}\left(\sum_{k=1}^{K} c_{k}\right),
$$

where the demand of NSP $k \in \mathcal{K}$ is $c_{k}$. The set of demands of all NSPs are $\mathbb{C}:=\left\{c_{1}, c_{2}, \ldots, c_{K}\right\}$. In this work, we consider $c_{k} \in \mathbb{C}$ is the spectrum demand based on the users' selection of services.

We also denote the price strategy of SCP is $\boldsymbol{v}=\left\{v_{0}, v_{1}\right\}$ where $v_{0} \in \boldsymbol{R}_{+}$is the pricing vector according to NSPs' demand. $v_{1}$ is a constant and we consider $v_{1} \geq 1$ so that payoff is a convex function [16]. The revenue of SCP depends on its price strategy as well as the request of NSPs. The payoff of SCP is the difference between its revenue and cost, which is given as

$$
\varpi(\boldsymbol{e}, \boldsymbol{v}, \mathbb{C})=\sum_{k=1}^{K} v_{0}\left(c_{k}\right)^{v_{1}}-E(\boldsymbol{e}, \mathbb{C}) .
$$

2) Payoff of NSP: In the upper-level game, the profit of a NSP also consists of its revenue and cost. The revenue is obtained by serving users who select its services and the cost is from the charges of renting resources supplied by SCP. Therefore, the payoff of NSP $k$ can obtain is denoted as

$$
\pi_{k}(\mathbb{C})=\Gamma_{k}-v_{0} c_{k}^{v_{1}},
$$

where $\Gamma_{k}$ is the revenue of NSP $k$. We can assume that $\Gamma_{k}=\gamma \beta_{k} c_{k}$, where $\gamma$ is the spectrum efficiency that SCP can provide and $\beta_{k}$ is the revenue unit of data rates. We further denote that $c_{-k}$ denote the set of strategies adopted by all except NSP $k$ and the strategies set $\mathbb{C}$, i.e., $\mathcal{C}=c_{-k} \cup c_{k}$.

Remark 1: We have given different notions in (2) and (9) to present the payoff of NSPs in different levels. This is due to the fact that the considerations of NSPs in two decision making process are different.

Remark 2: The revenues of NSPs can be different in (2) and (9). Nevertheless, in order to provide guaranteed services to the users, $\gamma c_{k}$ should be no less than the summation of the users' requirements to NSP $k$.

\section{B. Monopoly Market of SCs}

In this part, we consider there is only one SCP in the system and all NSPs needs to compete with each others in terms of amount of SBSs supplied to the market to gain the maximum payoff. First, the SCP announces its pricing policies. According to the SCP's announcement and users' subscriptions, NSPs make the best responses and then decide the renting policies. A Stackelberg game is used to analyze this situation and the Stackelberg equilibrium (SE) is considered as the solution of this game. A static game is formulated and Nash equilibrium is obtained in a centralized manner. In this case, the optimal rent policy to one SCP depends on the strategies of NSPs. SE is considered as the solution of the game and it is defined as

Definition 3. The strategy profile of upper-level game is a SE if all parties in the monopoly market, i.e. SCP and NSPs, $v$ and $\mathbb{C}$ are optimal strategies given others' strategies.

Given the leasing decision of NSPs, for SCP, the optimal pricing policy is

$$
\boldsymbol{v}^{*}=\arg \max _{\boldsymbol{v}} \varpi(\boldsymbol{v})
$$

The price of SCP involves the leasing policy of NSPs as well as its cost function. In the NSPs' game, the SPE is obtained by using the best response function which is the best strategy of one player given others' strategies. The best response function of NSP $k$ given the strategies of other NSPs, is defined as follows:

$$
\mathcal{B}\left(c_{-k}\right)=\arg \max _{c_{k}} \pi_{k}\left(c_{k}, c_{-k}\right) .
$$

The set $\mathcal{C}^{*}=\left\{c_{1}^{*}, c_{2}^{*}, \ldots, c_{K}^{*}\right\}$ is the Nash equilibrium of the formulated game iff for

$$
c_{k}^{*}=\mathcal{B}\left(c_{-k}^{*}\right), \forall k .
$$

In order to obtain Nash equilibrium, the following set of equations can be solved,

$$
\frac{\partial \pi_{k}(\mathbb{C})}{\partial c_{k}}=0 .
$$

A numerical method can be used to obtain the SE by solving (13). Alternatively, we formulate an optimization problem that minimize the sum of the differences between decision variables $c_{k}$ and the corresponding best response functions. Note that the minimum value of the objective is zero if the algorithm reaches the SE.

\section{Performance Evaluations}

First, we consider a simply example (Case 1) that there are two NSPs and each of NSP has only one services, i.e. $|\mathcal{K}|=2$ and $S_{k}=1, \forall k \in \mathcal{K}$ to simply illustrate the proposed scheme. In addition, we assume $U_{y}(\boldsymbol{d})$ in (1) can be expressed as follows, $U_{1}(\boldsymbol{d})=\alpha\left(\zeta_{1}-3.5 d_{1}\right)-p_{1}, U_{2}(\boldsymbol{d})=\alpha\left(\zeta_{2}-\right.$ $\left.4 d_{2}\right)-p_{2}$. We also assume $v_{1}=2, N=200$, and $\alpha=1$ for simplicity. NSPs and SCP vary their strategies, i.e., $\mathcal{P}$ and $v_{0}$ to maximize their payoffs. $\zeta_{k}, k=1,2$ is the maximum QoS that service provider can offer. In the simulations, we consider SCP is able to guarantee a constant SNR or spectrum efficiency so the spectrum demand of NSP only depends on the number of served users and their selected services. 


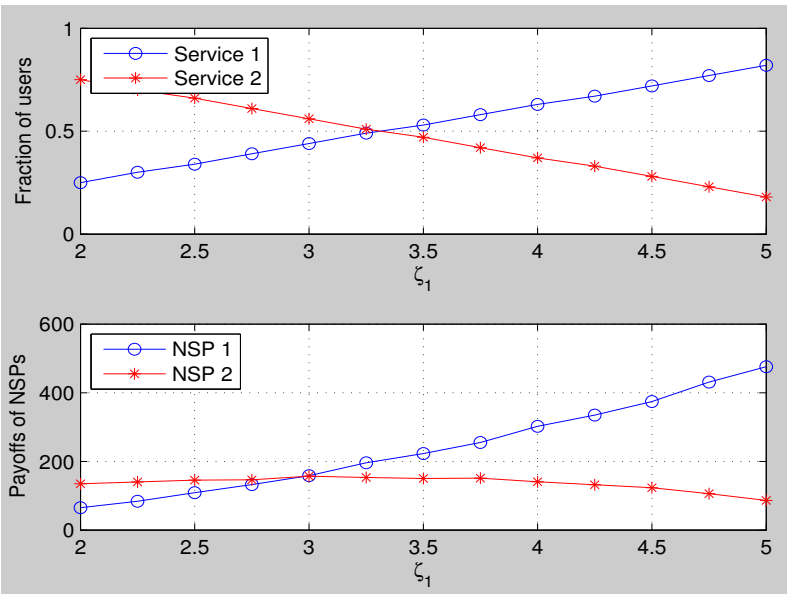

Fig. 2. a) Fraction of users and b) Payoffs of NSPs in Case 1

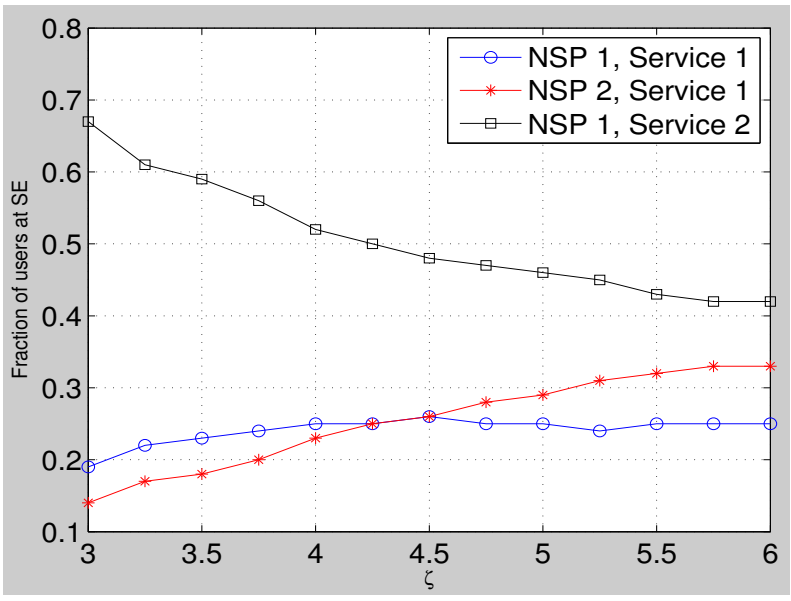

Fig. 3. Fraction of users of services and NSPs in Case 2

In Fig. 2, we fix $\zeta_{2}=3$ and vary $\zeta_{1}$ to see its impact on the performance. Due to the fact that the increment of $\zeta_{1}$ indicates that NSP 1 is able to host more users, as $\zeta_{1}$ increase, more and more users start to transfer from service 2 to service 1 at $\mathrm{SE}$, which is shown in Fig. 2 a). However, due to the pricing strategies and the the users who remain to choose service 2 can get increased payoff, the payoff of the NSP 2 only decreased slightly as shown in Fig. 2 b).

In Fig. 3, we assume that NSP 1 can offer two services (Service 1 and 2) and NSP 2 has only one (Case 2). The $U_{y}(\boldsymbol{d})$ can be simplified as as, $U_{1}(\boldsymbol{d})=4-2.5 d_{1}-p_{1}, U_{2}(\boldsymbol{d})=$ $\zeta-2.8 d_{2}-p_{2}$ and $U_{3}(\boldsymbol{d})=3-1.5 d_{3}-p_{3}$. We consider service 2 is offered by NSP $s$ and also vary $\zeta$ to see its impact on the performance. Similarly, the the increment of $\zeta$ can increase the QoS capabilities of NSP 2 as $\zeta$ gets larger, more and more users start to transfer from NSP 1 to NSP 2 at SE, which is shown in Fig. 2. This is mainly due to the fact that users who subscribe to NSP 2 can obtain better payoffs. It can also be noticed the Service 1 also attract slightly more users.

\section{CONCLUSION}

In this work, we have proposed a game theoretical approach to address the interactions among users, NSPs and SCP in the small cell networks. Specifically, we have considered that the NSPs need to lease from the SCP in order to provide multiple services to a number of users based on the users' requests. To model this interactive decision problem, a hierarchical game framework is presented. In the lower-level, a multileader multi-follower Stackelberg game is formulated to model and analyze the adaptive service selection of non-atomic users and service pricing of NSPs. In the upper-level, the NSPs and $\mathrm{SCP}$ sequentially determine the leasing and pricing strategies, respectively, by taking into account the service selection in the lower-level game. Performance evaluation shows the effectiveness and advantages of the proposed approach.

\section{ACKNOWLEDGEMENT}

This work is partially supported by the Academy of Finland (Decision No. 284748, 288473).

\section{REFERENCES}

[1] P. Lin, J. Zhang, Y. Chen, and Q. Zhang, "Macro-femto heterogeneous network deployment and management: From business models to technical solutions," IEEE Wireless Commun., vol. 18, no. 3, Jun. 2011.

[2] T. Q. S. Quek, G. de la Roche, I. Guvenc, and M. Kountouris, Small Cell Networks: Deployment, PHY Techniques, and Resource Management. Cambridge University Press, 2013.

[3] D. Niyato and E. Hossain, "A game theoretic analysis of service competition and pricing in heterogeneous wireless access networks," IEEE Trans. Wireless Commun., vol. 7, no. 12, pp. 5150-5155, Dec. 2008.

[4] S. Sengupta and M. Chatterjee, "An economic framework for dynamic spectrum access and service pricing," IEEE/ACM Transactions on Networking, vol. 17, no. 4, pp. 1200-1213, August 2009.

[5] L. Rose, E. V. Belmega, W. Saad, and M. Debbah, "Pricing in heterogenous wireless networks: hierarchical games and dynamics," IEEE Trans. Wireless Commun., vol. 13, no. 9, pp. 4985-5001, Sep. 2014.

[6] Y. Yi, J. Zhang, Q. Zhang, and T. Jiang, "Spectrum leasing to femto service provider with hybrid access," Proc. IEEE INFOCOM, Orlando, FL, USA, 2012.

[7] Y. Chen, J. Zhang, and Q. Zhang, "Utility-aware refunding framework for hybrid access femtocell network," IEEE Trans. Wireless Commun., vol. 11, no. 5, pp. 1688-1697, May 2012.

[8] S. Ren, J. Park, and M. van der Schaar, "Entry and spectrum sharing scheme selection in femtocell communications markets," IEEE/ACM Trans. Netw., vol. 21, no. 1, pp. 218-232, Feb. 2013.

[9] X. Kang, R. Zhang, and M. Motani, "Price-based resource allocation for spectrum-sharing femtocell networks: a Stackelberg game approach," IEEE J. Sel. Areas Commun., vol. 30, no. 3, pp. 538-549, Apr. 2012.

[10] K. Zhu, E. Hossain, and D. Niyato, "Pricing, spectrum sharing, and service selection in two-tier small cell networks: a hierarchical dynamic game approach," IEEE Trans. on Mobile Comp., vol. 13, no. 8, pp. 18431856, July 2014.

[11] J. G. Wardrop, "Some theoretical aspects of road traffic research," Proceedings of the Institute of Civil Engineers , 1952.

[12] David Schmeidler, "Equilibrium points of non-atomic games", Journal of Statistical Physics, Vol. 7, 295-301, 1973.

[13] A. de Palma, and Y. Nesterov, "Optimization Formulations and Static Equilibrium in Congested Transportation Networks," CORE Discussion Papers 9861, Universite Catholique de Louvain, Louvain-la-Neuve, Belgium, 1998.

[14] M. Beckmann, C. B. McGuice, and C. Winsten, Studies on the Economics of Transpoortations, Yale University press, New Haven, CT, 1956.

[15] V. Berinde, Iterative Approximation of Fixed Points, 2nd Edition, Springer-Verlag, 2007.

[16] D. Niyato and E. Hossain, "Competitive spectrum sharing in cognitive radio networks: a dynamics game approach," IEEE Transactions on Wireless Communications, vol. 7, no. 7, July 2008. 